\title{
The Design Of A New Phased Array Antenna For S-Band
}

\author{
Tao YUAN, Ji-Lan LI, Xin ZHANG \\ School of Electronic Information Engineering , Wuyi University, Jiangmen Guangdong 529020, China
}

Keywords: Quasi-Yagi antenna; Impedance bandwidth; Pattern; Microstrip antenna

\begin{abstract}
The design of a new phased array antenna for S-band is designed in this paper. The structure of the new antenna is based on the traditional microstrip quasi-Yagi antenna. The $\mathrm{G}$ type micro-strip line is designed on the back of the antenna for feed line. Two director elements and two driver elements are increased to improve the gain. The top of the director elements is grooved to expand the bandwidth. This antenna is designed and analyzed by comparing with simulation software and antenna test. The results of the modified quasiYagi show a relative bandwidth about $40 \%$ for Voltage Standing Wave Ratio(VSWR) of less than 2 and the maximum gain about $6 \mathrm{~dB}$ at center frequency $2.4 \mathrm{GHz}$. The method of reasonable structure design is testified can effectively improve the bandwidth and gain of this antenna. It has good application prospects.
\end{abstract}

\section{Introduction}

With the development of science and technology, phased array antennas are often required to obtain large instantaneous bandwidth in wide angle scanning. Due to the aperture effect and the aperture transit time, not only the instantaneous bandwidth signal is limited, but also the beam pointing of the antenna is offset. In order to eliminate the influence of these two defects, the optical fiber delay line in each cell and each sub array can be used to eliminate the aperture transit time [1]. Microstrip antennas are widely used in radar, reconnaissance, navigation and mobile communications [2], so people have higher requirements on the bandwidth and gain of the antenna. However, microstrip antenna has a narrow bandwidth, so how to improve the performance has become a hot research topic [2].

The quasi Yagi antenna proposed by Qian et al, inherited the radiation characteristics of the tradition Yagi antenna [3]. The size of this antenna was designed very small by using dielectric material with high dielectric constant. It is easy to form antenna array. The structure of the traditional quasiYagi antenna was designed and improved in this paper, in order to improve the antenna gain and relative bandwidth [4].

The antenna of paper [2] based on the traditional quasi-Yagi antenna has the characteristics of broadband and wide angle by using double micro-strip patches instead the double arm oscillators of the quasi-Yagi antenna. In paper [3], it makes full use of quasi-Yagi antenna space. Two parasitic patches and oscillator are increased in order to improve the relative bandwidth. The director dipole is added to improve the gain of the antenna. In paper [3], the micro-strip patch antenna array is designed to enhance bandwidth and gain. In paper [5],the microstrip patch antenna array is designed to enhance the bandwidth and gain. In paper [6], the performance of micro-strip Yagi patch antenna depends on the coupling between intensity and phase delay is verified by experiments. In paper [7], it was proposed and verified that the size and position of the director are the main factor that affect the gain of the antenna. In paper [8], it was confirmed that the distance between the double arm oscillators of the quasi-Yagi antenna have influence on the insertion loss. The antenna of this paper was based on the above documents to improve the gain and the bandwidth of the antenna.

\section{The antenna design}

In this paper, the length and width of balun oscillator was changed in order to improve the bandwidth of the quasi Yagi microstrip antenna. The G type micro-strip line is designed on the back of the antenna to change the beam width. Two director elements and two driver elements are increased to improve the gain. The top of the director elements is grooved to expand the bandwidth. Then a center frequency of $2.4 \mathrm{GHz}$ new type quasi Yagi microstrip antenna for phased array antenna was designed. In order to realize the miniaturization of the antenna, the dielectric plate ROGER of the high frequency plate is improved to FR4 of the epoxy resin plate, the dielectric constant is changed from 2.2 to 4.4 , and the thickness of the plate is $1.6 \mathrm{~mm}$. Fig. 1 shows the structure of the unit antenna, Fig. 2 is the element antenna. 


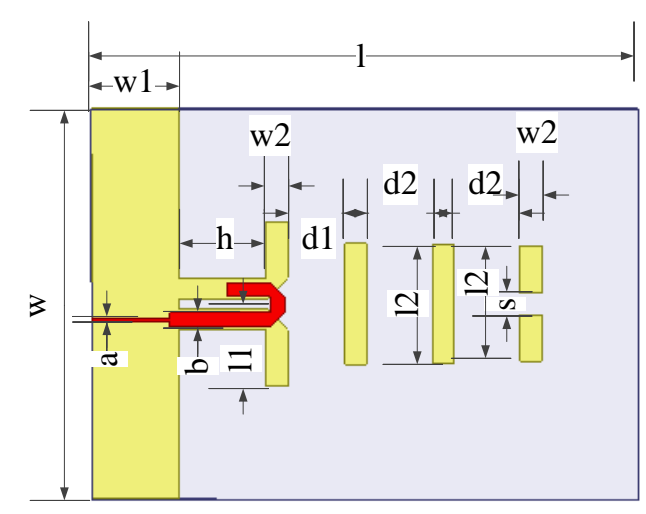

Fig.1 The structure of the antenna.

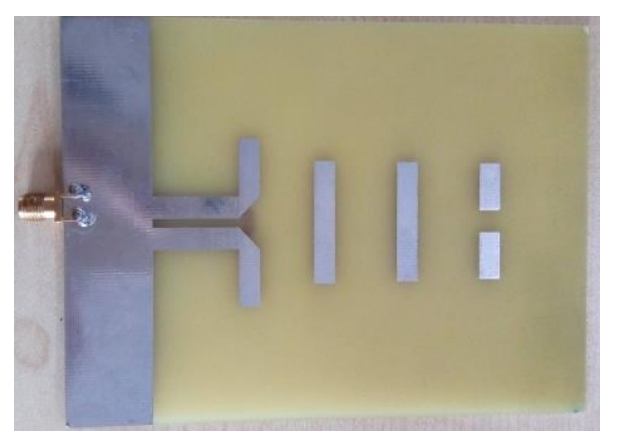

(a) The front of the antenna.

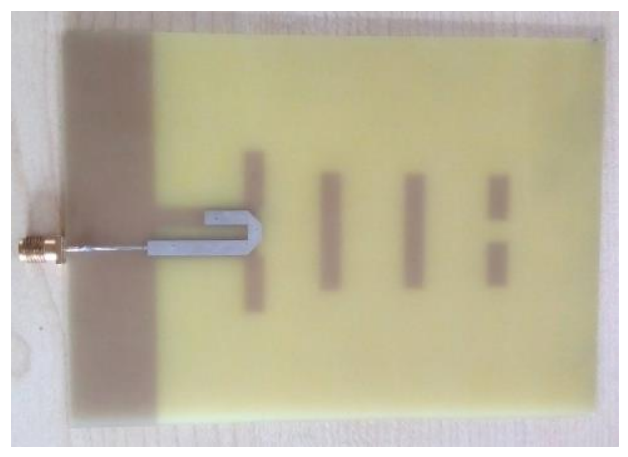

(b) The back of the antenna.

Fig. 2 The element antenna.

According to the design based on the quasi Yagi antenna, the length of the oscillator is $0.5 \lambda_{g}$, the length of the director is $0.45 \lambda_{g}$. Where $\lambda_{g}$ is the effective wavelength for the antenna and

$$
\lambda_{g}=\frac{c \sqrt{2}}{f_{0} \sqrt{\varepsilon_{r}+1}}
$$

The equivalent relative dielectric constant $\varepsilon_{e}$ is

$$
\varepsilon_{e}=\frac{\varepsilon_{r}+1}{2}+\frac{\varepsilon_{r}-1}{2}\left(1+10 \frac{h}{w}\right)^{-\frac{1}{2}}
$$

Where $c$ is the speed of light in free space, $f_{0}$ is the center frequency, $\varepsilon_{r}$ is the relative dielectric constant, $h$ is the thickness of dielectric plate, $w$ is the width of the microstrip line.

$$
w=\frac{c}{f_{r} \sqrt{2\left(\varepsilon_{r}+1\right)}}
$$

Where $f_{r}$ is the resonant frequency of the antenna.

Using the above formula, we can get the initial value of each parameter, and then we can get the parameters of the antenna by using Ansoft HFSS simulation software: $w=90 \mathrm{~mm}$, $1=125 \mathrm{~mm}, \quad w 1=20 \mathrm{~mm}, \quad w 2=5 \mathrm{~mm}, \quad \mathrm{~s}=5 \mathrm{~mm}, \quad 11=26 \mathrm{~mm}$, $12=28 \mathrm{~mm}, \quad d 1=13 \mathrm{~mm}, \quad \mathrm{~d} 2=20 \mathrm{~mm}, \mathrm{~h}=20 \mathrm{~mm}, \quad b=3.4 \mathrm{~mm}$, $\mathrm{a}=0.7 \mathrm{~mm}$.

\section{Simulation and analysis}

\subsection{Parameter selection}

Microstrip quasi Yagi antenna is composed of balun, radiation units and director. The bandwidth is mainly decided by the form of balun and oscillator, we can optimize the position, width and length of the oscillator to broaden bandwidth. For microstrip quasi Yagi antenna excitation oscillator length mainly affects the resonant frequency, the distance between the excited oscillator and the reflector is mainly affected by the surface electric field distribution, which affects the gain and matching.

We mainly consider the influence of oscillator width $w 2$, the distance between the excited oscillator and the reflector $h$, the length of the excited oscillator $l 1$ and the gap of the director $S$.

(1) Analysis the influence of the width of the oscillator $w 2$. When the width of the oscillator $w 2$ was $4 \mathrm{~mm}, 5 \mathrm{~mm}$, and $6 \mathrm{~mm}$, the S11 curve is shown in Fig. 3. From Fig. 3, with the increase of the width of the time, the return loss value and bandwidth is basically unchanged, but the resonant frequency decreases, $w 2$ has great influence on the antenna resonant frequency. 


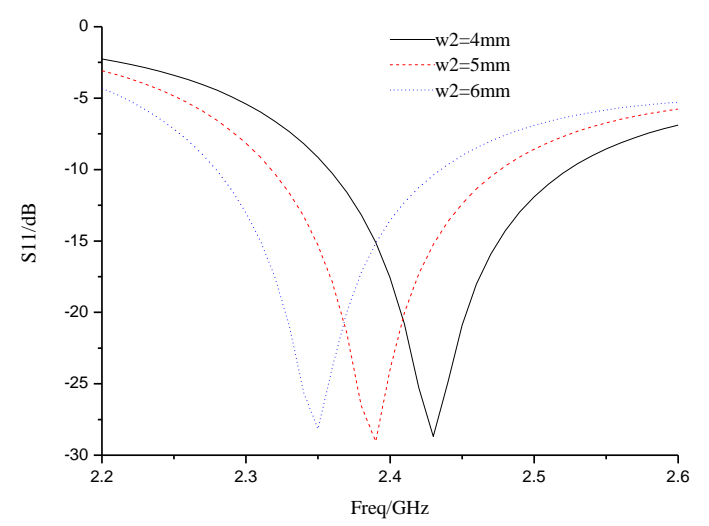

Fig.3 The influence of $w 2$ on S11.

(2) Analysis the influence of the distance between excitation oscillator and director oscillator $h$. When $h$ was $18 \mathrm{~mm}$, $20 \mathrm{~mm}$ and $22 \mathrm{~mm}$, the $\mathrm{S} 11$ curve is shown in Fig. 4. From Fig. 4 , With the increase of $h$, the resonant frequency becomes smaller, when $h=20 \mathrm{~mm}$, the return loss and the bandwidth of the antenna are optimized, and the matching characteristic of the antenna is changed, whether $h$ increases or decreases. The distance between excitation oscillator and director oscillator $h$ has an effect on the antenna resonant frequency and matching characteristic.

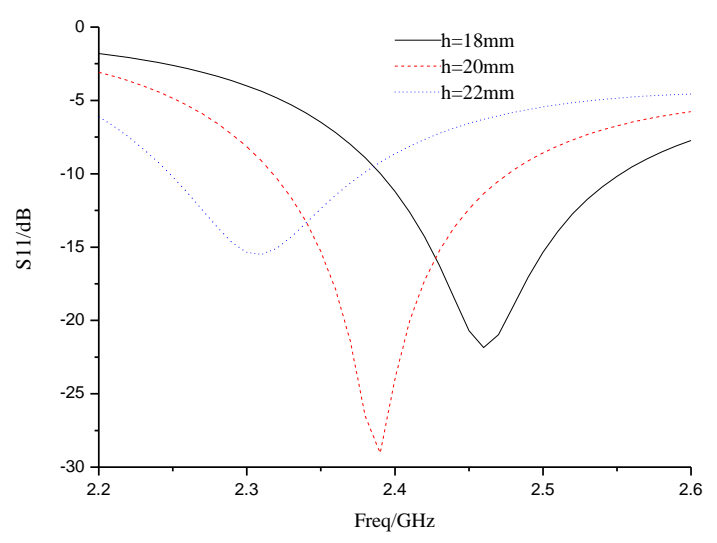

Fig.4 The influence of $h$ on S11.

(3) Analysis the influence of the length of the excitation oscillator $l 1$. When the length of the excitation oscillator $l 1$ was $24 \mathrm{~mm} 、 26 \mathrm{~mm}$ and $28 \mathrm{~mm}$, the $\mathrm{S} 11$ curve is shown in Fig. 5. It can be seen from Fig.5, with the increase of the distance, the resonant frequency decreases, and the matching characteristics become better, when $l 1=26 \mathrm{~mm}$, the return loss and the bandwidth of the antenna are optimized. The length of excitation oscillator $l 1$ has an effect on the antenna resonant frequency and matching characteristic.

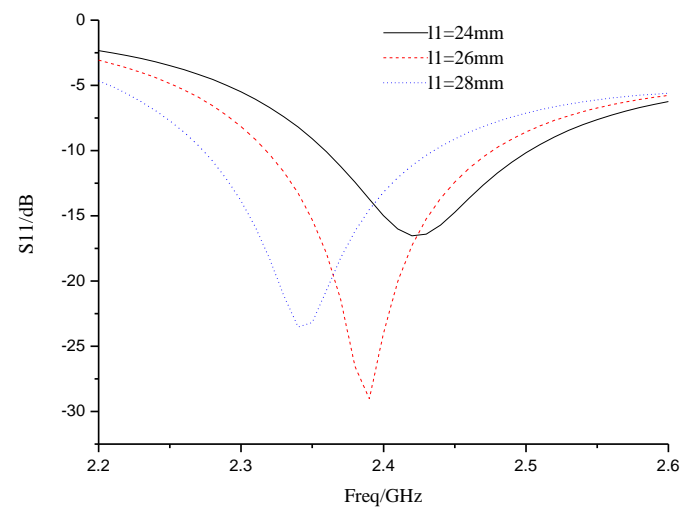

Fig. 5 The influence of $l 1$ on $\mathrm{S} 11$.

(4) Analysis the influence of the gap of the director $s$. When the gap of the director $s$ was $4 \mathrm{~mm}, 5 \mathrm{~mm}$ and $6 \mathrm{~mm}$, the $S 11$ curve is shown in Fig. 6. It can be seen from Fig.6, while little change of the gap of the director $s$, it has not much affect on the resonance frequency and the bandwidth of the antenna.

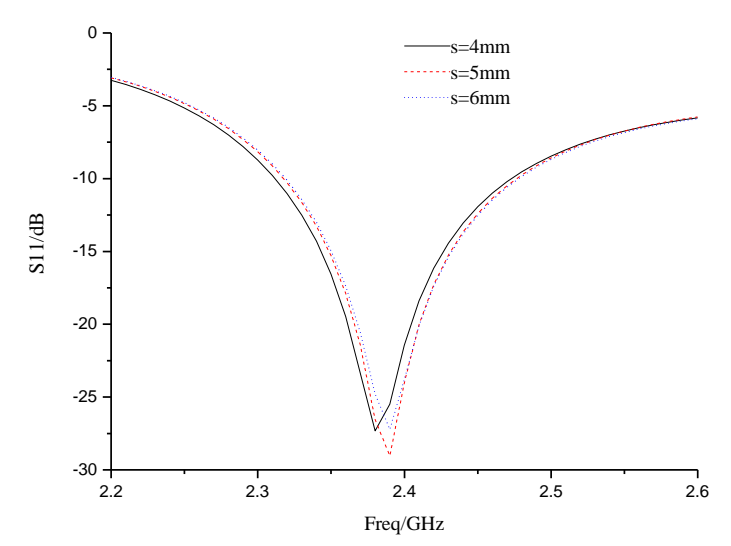

Fig.6 The influence of $s$ on S11.

\subsection{Comparative analysis of results}

Comparing the measured results with the simulation results, it can be seen that the two are in general agreement, but there are some deviations. The measured resonant frequency is offset and the bandwidth is relatively small. The accuracy of the antenna processing, the welding technology of the SMA joint and the quality of the dielectric substrate are all the reasons for these deviations.

The S11 parameter of the simulation and measurement is shown in Fig.7. As can be seen from Fig.7, both the relative bandwidth of $S 11 \leq-10 \mathrm{~dB}$ are $40 \%$. The voltage standingwave ratio (VSWR) of the simulation and measurement is shown in Fig.8. As can be seen from Fig.8, both the relative bandwidth of $V S W R \leq 2$ are $42 \%$. The gain of the simulation and measurement is shown in Fig.9. As can be 
seen from Fig.9, both the gain of the antenna are more than $6 \mathrm{~dB}$, when the center frequency is $2.4 \mathrm{GHz}$.

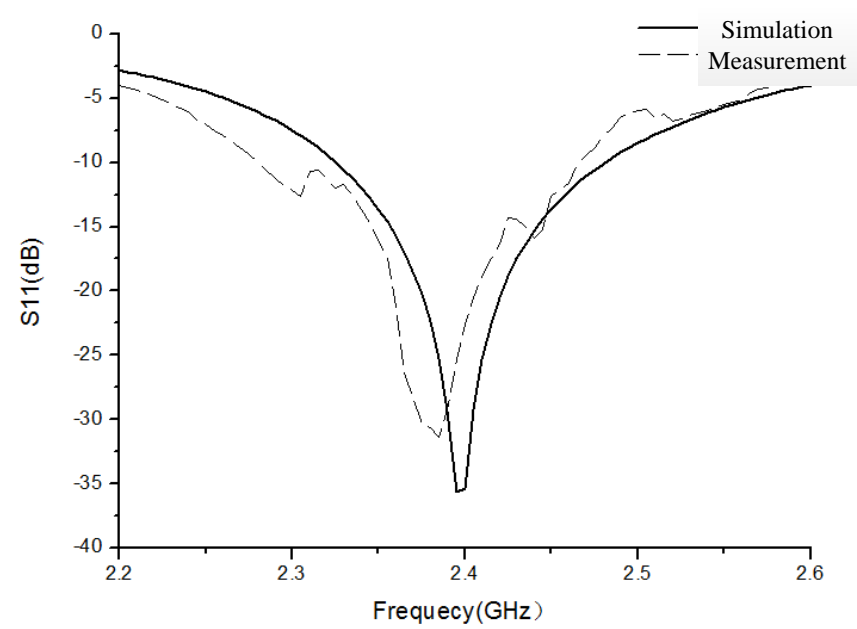

Fig.7 The S11 parameter result.

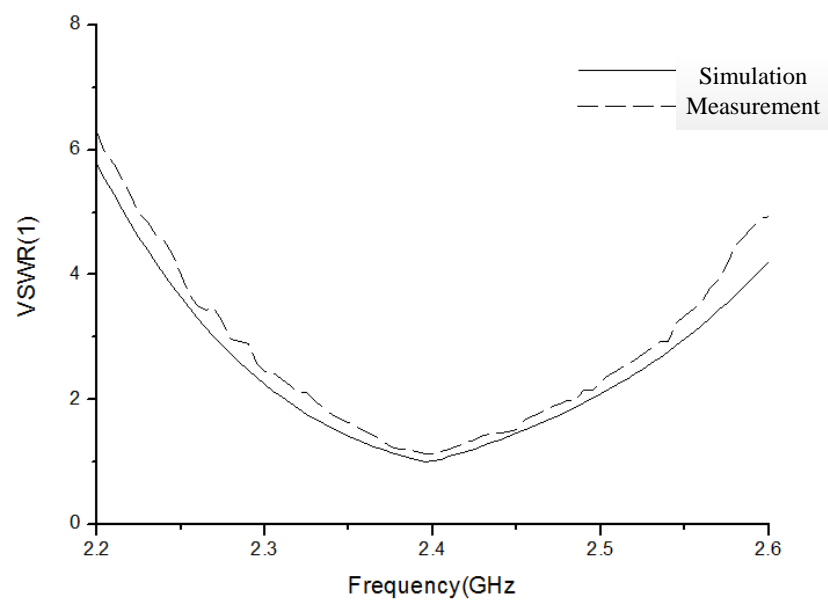

Fig.8 The VSWR result.

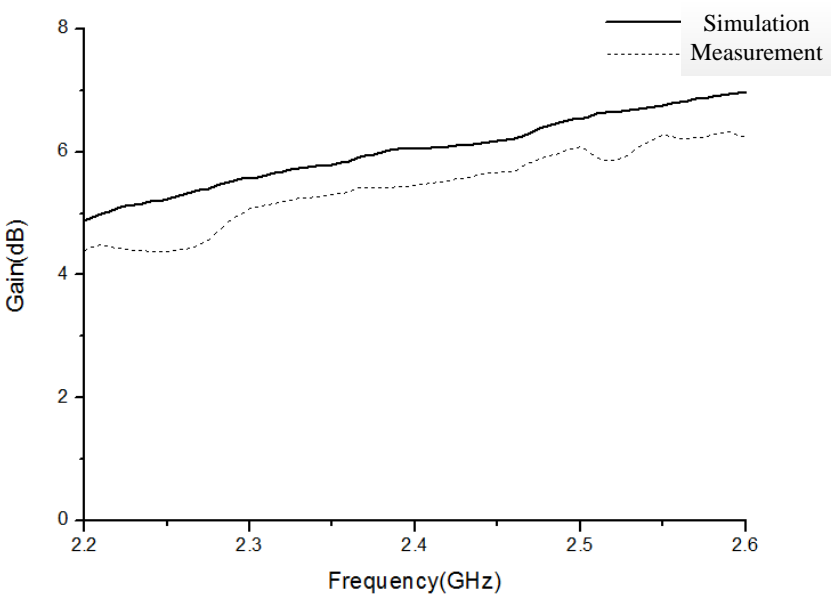

Fig.9 The gain result.
Comparing the results of simulation and measurement is shown in table 1 , from table 1 , both results are more or less alike. It confirmed the rationality of the design.

\begin{tabular}{|c|c|c|c|}
\hline & $\begin{array}{l}\text { gain } \\
(\mathrm{dB})\end{array}$ & $\begin{array}{l}\text { Relative ban } \\
V S W R \leq 2\end{array}$ & $\begin{array}{l}\text { dwidth } \\
S 11 \leq-10 \mathrm{~dB}\end{array}$ \\
\hline simulation & 6.0439 & $42.5 \%$ & $40 \%$ \\
\hline measurement & 5.4574 & $40.8 \%$ & $38 \%$ \\
\hline
\end{tabular}

Table1: Comparative the simulation and measurement results.

\section{Conclusion}

The structure of the new antenna is based on the traditional microstrip quasi-Yagi antenna. The $\mathrm{G}$ type micro-strip line is designed on the back of the antenna for feed line. Two director elements and two driver elements are increased to improve the gain. The top of the director elements is grooved to expand the bandwidth. The results of the modified quasiYagi show a relative bandwidth about $40 \%$ for Voltage Standing Wave Ratio (VSWR) of less than 2 and the maximum gain about $6 \mathrm{~dB}$ at center frequency $2.4 \mathrm{GHz}$. The method of reasonable structure design is testified can effectively improve the bandwidth and gain of this antenna. It has good application prospects.

\section{Acknowledgements}

This work was supported by 2014 the Education Department of Guangdong Provincial special innovation projects No.2014KTSCX134.

\section{References}

[1] G.Y. Zhang. "Phased array radar system", National Defence Industry Press, (1994).

[2] C.F. She, B. Li, "A novel quasi-Yagi antenna with broadband and broad-angle characteristics", INFORMATION AND ELECTRONIC ENGINEERING, Vo1.9, No.1, pp.39-43, (2011).

[3] G.Q. Ni, T. Zhang, W. Ni. "Amelioration of the Quasi Yagi Antenna”, JOURNAL OF MICROWAVES, Vol.29, No.1,pp.51-54,(2011).

[4] J. Guo, F. Wang, M.P. Jin. "Design of a Microstrip-Fed Quasi-Yagi Antenna with Wide Bandwidth", Modern Electronics, vol. 79, No.2, pp. 25-27, (2002).

[5] B.H. Hou, G.B. Wan, X.S. Zhang, "Research on Characteristics of Microstrip Yagi Array Antenna", GHINESE SPACE SCIENCE AND TECHNOLOGY, vol.3, (2009).

[6] P. Wang, W.H. Chen, Z.H. Feng. National antenna Annual Conference: "Design of a Microstrip-Fed QuasiYagi Antenna withWide Bandwidth and High Gain", (2009). 
[7] Sharma P, Gupta S. "Bandwidth and gain enhancement in microstrip antenna array for $8 \mathrm{GHz}$ frequency applications", Students Conference on Engineering and Systems, pp. 1-6, (2014).

[8] Mitola J, Maguire G, Jr. "Cognitive radio: Making softwave radios more personal", IEEE Personal Communication Magazine, Vol.6, No.4, pp.13-18, (1999).

[9] Qian Y,Deal W R,Kaneda N,et al. "Microstrip-fed quasiYagi antenna with broadband characteristics", Electronics Letters,Vol.34,No.23, pp.2194-2196,(1998).

[10] Dandawate A V, Giannakis G B. "Statistical test for presence of cyclostationarity", IEEE trans. on Signal Processing, Vol.42, No.9, pp.2355-2369, (1996). 